\title{
Gastrectomía laparoscópica en manga para el tratamiento de lipoma gástrico gigante en paciente con obesidad mórbida
}

\author{
Laparoscopic sleeve gastrectomy for giant gastric lipoma in morbid obese patient
}

\author{
Rebeca Domínguez-Profeta, Robert F. Cubas*, Ryan Broderick, Arielle Lee, \\ Joslin Cheverie y Santiago Horgan \\ División de Cirugía Mínimamente Invasiva, University of California, San Diego, California, Estados Unidos
}

\begin{abstract}
Resumen
Antecedentes: Los lipomas gástricos corresponden al 5\% de los lipomas gastrointestinales. Muchos de ellos son solitarios, pequeños y asintomáticos, pero también pueden ocasionar síntomas obstructivos y sangrados. El tratamiento estándar es quirúrgico. Caso clínico: Mujer de 50 años con antecedente de obesidad mórbida, índice de masa corporal de $47.4 \mathrm{~kg} / \mathrm{m}^{2}$, que se presenta con síntomas de epigastralgia y anemia. Se diagnostica un lipoma gástrico de $6.3 \mathrm{~cm}$ mediante tomografía y se confirma por biopsia endoscópica. Discusión: La paciente fue exitosamente tratada a través de gastrectomía laparoscópica en manga. Conclusión: La gastrectomía laparoscópica en manga es el procedimiento de elección para el tratamiento de los lipomas gástricos gigantes en los pacientes con obesidad mórbida cuando la anatomía lo permite.
\end{abstract}

Palabras clave: Lipoma gástrico. Manga gástrica. Obesidad mórbida.

\begin{abstract}
Background: Gastric lipomas account for $5 \%$ of all gastrointestinal lipomas. Most of them are solitary, small and asymptomatic, however, they can cause severe symptoms such as obstruction, bleeding and intussusception. The standard treatment is surgical resection. Case report: 50 years old female with history of morbid obesity with a body mass index (BMI) of $47.4 \mathrm{Kg} /$ $\mathrm{m2}$, who presented with symptoms of epigastric pain and anemia. CT scan of the abdomen revealed a $6.3 \mathrm{~cm}$ gastric lipoma, confirmed by endoscopic biopsy. Discussion: Laparoscopic sleeve gastrectomy is the procedure of choice for the excision of giant gastric lipomas in the morbidly obese, when anatomically feasible.
\end{abstract}

Key words: Gastric lipoma. Sleeve gastrectomy. Morbid obesity.

\section{Introducción}

Los lipomas del tracto gastrointestinal son tumores mesenquimales benignos de aparición poco común. Los lipomas gástricos conforman alrededor del $5 \%$ de todos los lipomas del tracto gastrointestinal, y constituyen del 2 al $3 \%$ de todos los tumores gástricos benignos y menos del $1 \%$ de todas las neoplasias gástricas $^{1,2}$. La mayoría de los lipomas son de localización submucosa $(95 \%)$ y solo ocasionalmente

\section{Correspondencia:}

*Robert F. Cubas

Division of Minimally Invasive Surgery

University of California, San Diego

PO Box 232410

Fecha de recepción: 09-06-2019

Fecha de aceptación: 01-04-2020

San Diego, CA 92193, USA

E-mail: rfc57@miami.edu

DOI: 10.24875/CIRU.20001354
Cir Cir. 2020;88(S1):39-42 Contents available at PubMed www.cirugiaycirujanos.com (http://creativecommons.org/licenses/by-nc-nd/4.0/). 
subserosa (5\%). Un $75 \%$ de los casos son lesiones antrales solitarias, en su mayoría asintomáticas ${ }^{3,4}$. Pueden presentarse como un hallazgo incidental, pero pueden causar síntomas graves, como obstrucción, hemorragia e intususcepción ${ }^{5,6}$.

La impresión diagnóstica se realiza mediante tomografía computada abdominal y se confirmada con el estudio histopatológico. El tratamiento estándar es la resección quirúrgica, aunque se ha propuesto la vía endoscópica para tumores de pequeño tamaño1.

Presentamos el caso de una paciente con obesidad mórbida en la que se realizó una manga gástrica laparoscópica para la exéresis de un lipoma gástrico gigante.

\section{Caso clínico}

Mujer de 50 años con obesidad mórbida (índice de masa corporal $47.4 \mathrm{~kg} / \mathrm{m}^{2}$ ), diabetes mellitus controlada con dieta, hipotiroidismo, ansiedad y ataques de pánico, quien presenta múltiples episodios de epigastralgia, anemia microcítica $(8.4 \mathrm{~g} / \mathrm{dl})$ y tomografía computada de tórax que muestra la presencia de una masa gástrica lipomatosa, de $6.3 \times 3.2 \mathrm{~cm}$, localizada en la curvatura mayor del estómago. La endoscopia digestiva alta confirmó el hallazgo de una gran masa que se originaba en el cuerpo gástrico, en estrecha cercanía a la curvatura mayor, fácilmente deprimible, con eritema en ciertas áreas de la mucosa, pero sin lesiones aparentes (Fig. 1). Se realizó un ultrasonido endoscópico que mostró una masa subepitelial de 10 $\mathrm{cm}$. Se tomaron muestras para biopsia usando una aguja Procor de 22 Gauge. El resultado preliminar de la citología fue consistente con un tumor lipomatoso. Posteriormente se procedió a la toma de biopsias en túnel con forceps, en la que un tejido de apariencia lipomatosa protruyó a través de las áreas de las tomas de muestra. Al examen microscópico se evidenció tejido adiposo maduro consistente con lipoma. El esofagograma con trago de bario demostró una lesión lipomatosa gigante sin evidencia de obstrucción, y a su vez la tomografía computada abdominal mostró la presencia de una lesión adipomatosa a lo largo de la curvatura mayor con invaginación dentro del lumen, con medidas de $6.3 \times 3.8 \mathrm{~cm}$ y con densidad de 80 unidades Hounsfield (Fig. 2). Se planteó la resolución quirúrgica. Por la localización del lipoma y la obesidad mórbida de la paciente, se solicitó el consentimiento informado para proceder a la resección laparoscópica del tumor a través de una gastrectomía en manga, con el fin de proveer tratamiento definitivo para el

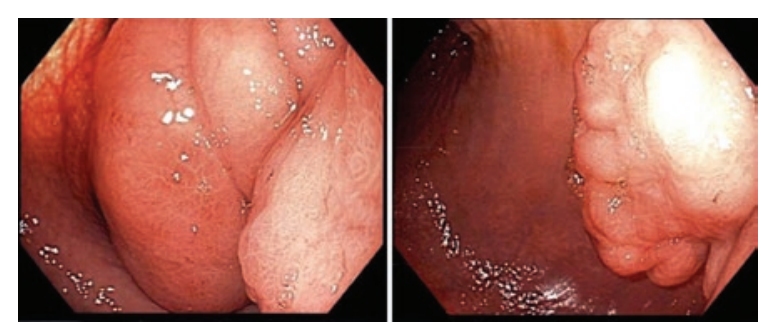

Figura 1. Endoscopía digestiva alta que muestra una masa lobulada en la curvatura mayor del estómago con mucosa conservada.

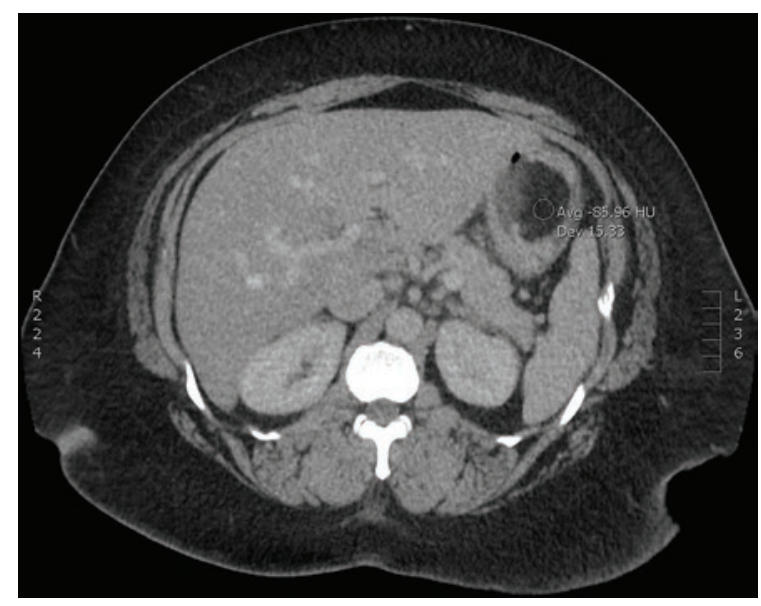

Figura 2. Tomografía computada del abdomen que muestra una masa gástrica de consistencia lipomatosa de $6.3 \times 3.2 \mathrm{~cm}$.

manejo de ambas patologías, tanto del tumor subepitelial gigante como de la obesidad.

La paciente fue trasladada al quirófano, se le colocaron medias de compresión neumática en ambas piernas y, tras la inducción de anestesia general e intubación endotraqueal, fue colocada en posición de semilitotomía. Se inició el procedimiento con una endoscopia digestiva alta, en la que se visualizó el tumor en la curvatura mayor sin evidencia de úlceras.

Se inició el neumoperitoneo a través de un puerto laparoscópico de $12 \mathrm{~mm}$ en la línea media en la región supraumbilical, posicionado bajo visión directa con una cámara de $0^{\circ}$, seguido de la inserción de tres trócares más para abordar el cuadrante superior izquierdo. Se posicionó a la paciente en Trendelemburg invertido. Se visualizó el tumor en la curvatura mayor del estómago. Los vasos gástricos cortos fueron divididos usando un dispositivo de energía avanzada. El fondo y el cuerpo del estómago fueron separados con extrema cautela de la cápsula anterior del páncreas. Se realizó una gastrotomía de $8 \mathrm{~cm}$ a lo largo de la curvatura mayor para 


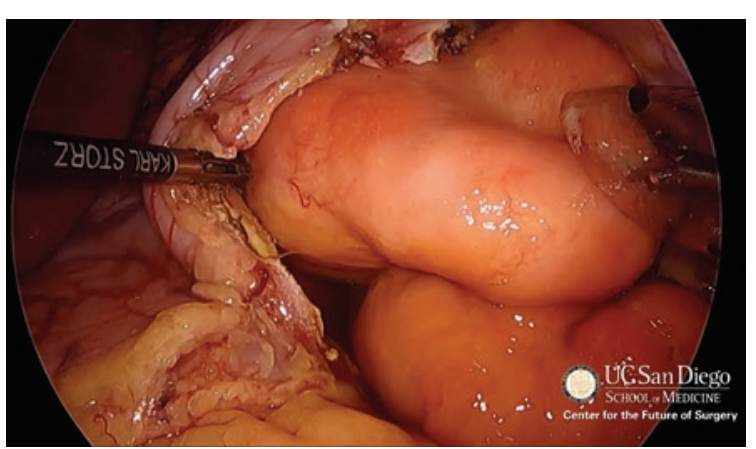

Figura 3. Imagen laparoscópica que muestra una masa lipomatosa gigante, expuesta después de dividir la curvatura mayor del estómago. La mucosa no presenta alteraciones.

exponer el tumor, utilizando un dispositivo bipolar para asegurar la hemostasia. La mucosa intragástrica no presentó evidencia de ulceraciones a la inspección, y después de exponer el tumor, este fue totalmente evertido a la cavidad peritoneal, traccionado con fórceps laparoscópico (Fig. 3) y seccionado usando tres cartuchos azules de $60 \mathrm{~mm}$ de engrapadora laparoscópica posicionada sobre la pared posterior a lo largo de la curvatura mayor. La pieza para anatomía patológica se colocó dentro de una endobolsa colectora. Después de confirmar la integridad de la mucosa gástrica, se suturó usando sutura barbada 2-0. La manga gástrica se llevó a cabo según la técnica estándar, realizando disparos secuenciales de engrapadora laparoscópica hasta el ángulo de His y utilizando una sonda calibradora de $36 \mathrm{Fr}$ conectada a succión.

Se realizó una endoscopia digestiva alta para evaluar la hemostasia de la línea de grapado. Se realizó la prueba hidroneumática, la cual resultó negativa. El tumor y el estómago fueron extraídos del abdomen a través de la incisión localizada en el cuadrante superior izquierdo. Se colocó un drenaje activo de Jackson Pratt a lo largo de la línea de grapado. Se retiraron los trócares y se cerró la piel con sutura intradérmica.

La histopatología describió una masa con medidas de $12.0 \times 11.5 \times 6.0 \mathrm{~cm}$ consistente con neoplasia lipomatosa benigna. No hubo complicaciones en el posoperatorio. Se indicó a la paciente dieta líquida a las pocas horas de la intervención. El primer día posterior a la cirugía se realizó un esofagograma con trago de bario en el que se evidenció una manga gástrica de anatomía apropiada, y como la paciente estuvo tolerando la dieta con líquidos claros sin dificultad se decidió su egreso. El drenaje fue retirado en el consultorio el día 7 posterior al procedimiento.

\section{Discusión}

Los lipomas gástricos son de rara presentación; constituyen menos del $1 \%$ de todos los tumores gástricos y la mayoría se originan en el antro (75\%). Los lipomas gástricos gigantes $(\geq 4 \mathrm{~cm})$ son de presentación extremadamente infrecuente 1 . Una revisión sistemática solo identificó 32 casos reportados desde $1980^{7}$. Los síntomas están directamente relacionados con el tamaño del lipoma: los $<2 \mathrm{~cm}$ a menudo permanecen asintomáticos, a diferencia de los de mayor tamaño $(>4 \mathrm{~cm})$, que pueden causar hemorragia, dispepsia, dolor abdominal y síntomas obstructivos ${ }^{2,3}$. Varios casos de pacientes obesos con tumores submucosos han sido reportados en la literatura, los cuales fueron detectados incidentalmente durante los estudios bariátricos preoperatorios o durante la cirugía ${ }^{1,8,9}$. En nuestro caso, el tumor tenía una medida de $6 \mathrm{~cm}$ y ocasionaba dolor abdominal y anemia microcítica. Considerando que la paciente presentaba obesidad mórbida, se tomó la decisión de tratar ambos problemas con un solo procedimiento quirúrgico. El tumor fue extirpado por completo antes de realizar la gastrectomía en manga para asegurar la indemnidad de la mucosa.

Los tumores sintomáticos requieren tratamiento quirúrgico, aunque el tratamiento de los lipomas gástricos continúa siendo un tema controvertido. Las lesiones $<3 \mathrm{~cm}$ y de ubicación submucosa se han eliminado eficazmente utilizando un lazo endoscópi$\mathrm{co}^{5}$. Las lesiones sintomáticas y $>3 \mathrm{~cm}$ requieren exéresis quirúrgica como tratamiento estándar ${ }^{5,6}$. Previamente a la intervención quirúrgica debe realizarse un detallado trabajo diagnostico que incluya tomografía y endoscopia alta con toma de biopsia. Asimismo, el uso de ecoendoscopia puede aportar imágenes para tomar muestras de biopsia más definitivas.

Si el diagnostico de lipoma se confirma, se puede intentar la resección endoscópica del lipoma y de la cápsula, y dejar las resecciones subtotales para tumores de mayor tamaño ${ }^{7}$. Otros autores han reportado diversas alternativas de resección, incluyendo enucleación local simple o escisión circunferencial con márgenes de tejido sano ${ }^{10}$.

La obesidad mórbida se asocia a un riesgo aumentado de neoplasias, entre las cuales los tumores del estroma gastrointestinal (GIST) tienen una mayor incidencia en la población obesa ${ }^{8}$. Durante una cirugía bariátrica puede identificarse un GIST de manera incidental, y en tal caso el tratamiento debe enfocarse en la resección del tumor con márgenes negativos ${ }^{1,8,11}$. 
En nuestra paciente, después de excluir la presencia de tumores que requerirían márgenes de resección más amplios, como el liposarcoma, se decidió realizar la gastrectomía en manga para resecar el tumor.

Hasta donde sabemos, este es el segundo caso reportado en la literatura de un tumor gástrico infrecuente resecado a través de gastrectomía laparoscópica en manga, y el primero que reporta esta técnica para el tratamiento de un lipoma gástrico gigante.

\section{Conclusión}

La gastrectomía laparoscópica en manga para la exéresis de lipomas gástricos gigantes en pacientes con obesidad mórbida es el procedimiento de elección cuando la anatomía lo permite. Este abordaje permite tratar eficazmente el tumor gástrico y el problema de obesidad del paciente. La utilización de endoscopia intraoperatoria es imperativa para evaluar la adecuada resección del tumor y la conformidad de la manga gástrica.

\section{Conflicto de intereses}

Los autores declaran que no existe conflicto de intereses.

\section{Responsabilidades éticas}

Protección de personas y animales. Los autores declaran que para esta investigación no se han realizado experimentos en seres humanos ni en animales.
Confidencialidad de los datos. Los autores declaran que han seguido los protocolos de su centro de trabajo sobre la publicación de datos de pacientes.

Derecho a la privacidad y consentimiento informado. Los autores han obtenido el consentimiento informado de los pacientes y/o sujetos referidos en el artículo. Este documento obra en poder del autor de correspondencia.

\section{Bibliografía}

1. Al Shamari J, Al-Shadidi N, Abdulsalam A, Al-Daihani A. Gastric lipoma excision during a laparoscopic sleeve gastrectomy: a case report. Int $J$ Surg Case Rep. 2016;24:128-30.

2. Fernandez F, Furian MC, Nascimento L, Amate A, Chubasi W, AgneIli BC, et al. Gastric lipoma presenting as a giant bulging mass in a oligosymptomatic patient: a case report. J Med Case Rep. 2012;6:317.

3. Reza JA, Frutcher S, Varadarajulu S, Amoletti JP. A large intussuscepting gastric lipoma. J Gastrointest Surg. 2018;7:1299-1300.

4. Termos S, Reslan O, Alqabandi O, Al-Dwaisan A, Al-Subaie S, Alyatama K, et al. Giant gastric lipoma presenting as a Gl bleed: enucleation or resection. Int J Surg Case Rep. 2017;41:3942.

5. Albert D, Grazioli L, Orizio P, Matricardi L, Dughi S, Gheza L, et al. Asymptomatic giant gastric lipoma: what to do? Am J Gastroenterol. 1999;12:3634-7.

6. Kibria R, Butt S, Ali SA, Akram S. An unusual case of giant gastric lipoma with hemorrhage. J Gastroenterol Cancer. 2009;40:144-5.

7. Cappell MS, Stevens CE, Amin M. Systematic review of giant gastric lipomas reported since 1980 and report of two new cases in a review of 117110 esophagogastroduodenoscopy. World J Gastroenterol. 2017;23:5619-33.

8. Walędziak M, Różańska-Walędziak A, Kowalewski PK, Janik MR, Brągoszewski J, Paśnik K. Bariatric surgery and incidental gastrointestinal stromal tumors: a single-center study. Wideochir Inne Tech Maloinwazyjne. 2017;12:325-9.

9. Crouthamel MR, Kaufman JA, Billing JP, Billing PS, Landerholm RW. Incidental gastric mesenchymal tumors identified during laparoscopic sleeve gastrectomy. Surg Obes Relat Dis. 2015;11:1025-8.

10. Hamndane MM, Brahim EB, Salah MB, Haouas N, Bouhafa A, Chedly-Debbiche A. Giant gastric lipoma mimicking well-differentiated liposarcoma. Pan Afr Med J. 2012;13:16.

11. Olguin RR, Norero ME, Briceno E, Martinez C, Vinuela E, Baez S, et al. Gastric lipoma removed by laparoscopic subtotal gastrectomy: report of one case. Rev Med Chil. 2013;141:927-31. 